\title{
A spectroscopic study of the eclipsing binaries SV Camelopardalis and XY Ursae Majoris`
}

\author{
F. F. Özeren ${ }^{1,3}$, A. G. Gunn ${ }^{2}$, J. G. Doyle ${ }^{1}$, and D. Jevremović ${ }^{1,4}$ \\ 1 Armagh Observatory, College Hill, Armagh BT61 9DG, N. Ireland \\ e-mail: jgd@star.arm.ac.uk \\ 2 University of Manchester, Jodrell Bank Observatory, Macclesfield, Cheshire, SK11 9DG, UK \\ e-mail: agg@jb.man.ac.uk \\ 3 Ankara University, Science Faculty, Astronomy and Space Sciences Dept., 06100 Tandog̃an-Ankara, Turkey \\ e-mail: ferhat@astro1.science.ankara.edu.tr \\ 4 Belgrade Observatory, Volgina 7, 11050 Belgrade, Yugoslavia \\ e-mail: darko@aob.aob.bg.ac.yu
}

Received 11 September 2000 / Accepted 9 November 2000

\begin{abstract}
High-resolution spectroscopic observations of the eclipsing RS CVn-type active close binaries SV Camelopardalis and XY Ursae Majoris reveal excess emission components in the $\mathrm{H} \alpha$ and $\mathrm{H} \beta$ lines. We have used the spectral subtraction technique to separate the excess emission from the photospheric background and find that in both stars the excess emission is associated with the secondary, cooler component. Analysis of the $E W_{\mathrm{H} \alpha} / E W_{\mathrm{H} \beta}$ ratio indicates that in both cases the emission is probably associated with surface plage regions or prominences viewed against the stellar disks. We find no evidence of excess absorption features associated with extended prominence-like material in these systems.
\end{abstract}

Key words. star: binaries: spectroscopic - stars: RS CVn, BY Dra binaries - stars: late type - stars: chromospheres of stars: magnetic fields

\section{Introduction}

Solar-like magnetic activity such as photospheric spots, chromospheric emission and flares are observed in stars of later spectral type. This is to be expected on the basis of the dynamo theory of magnetic field generation since such stars have substantial convective envelopes. In recent years observations have provided unexpected evidence for cool structures in the coronae of such active stars. Mullan et al. (1989) observed coronal condensations being ejected from the star V471 Tauri at projected line-of-sight velocities of up to $800 \mathrm{~km} \mathrm{~s}^{-1}$. Houdebine et al. (1990) reported a coronal mass ejection (CME) event during a flare on AD Leonis (dM4.5e). On rapidly rotating stars such as AB Doradus(dK0e), Gliese 890 (dM0e) and HK Aquarii (dM2e) cool material has been observed co-rotating in the stellar atmosphere (Collier Cameron et al. 1990; Doyle \& Collier Cameron 1990; Byrne et al. 1996).

The existence of these prominence-like structures on active stars has important consequences. Highly energetic ejections and the possibility of numerous coronal formations suggest that they could be a major component of the

Send offprint requests to: F. F. Özeren

* Based on observations made at Observatoire de Haute Provence (CNRS), France. stellar wind and mass loss into the ISM. However, the proportion of mass loss through discrete events and coronal thermal expansion remains to be established. A comparison of prominence-like structures amongst different types of active stars therefore has important implications for the solar paradigm.

The detection of cool material overlying the surface of a star is extremely difficult. However, in eclipsing binary systems (e.g. RS Canum Venaticorum-type binaries) the star occulted scans the corona of its companion easing the detection of the absorbing material. The technique of spectral subtraction, which involves a detailed comparison to "standard spectra", allows the disentanglement of the component spectral features. Hall et al. (1990) reported extensive observations of the RS CVn binary SS Bootis in the Balmer lines and Ca II H\&K. An unusual excess absorption was seen near primary conjunction which was attributed to an extended region of size $\sim 4 R_{\odot}$. Hall \& Ramsey (1992) surveyed 10 RS CVn systems and reported stable prominence-like material corotating in eight stars. This study concluded that amongst the eclipsing RS CVn stars, prominence material was a common feature. More recently Gunn \& Doyle (1997) and Gunn et al. (1997) questioned the existence of prominences in RS CVns although observations made with the 
Table 1. Parameters for the program and standard stars in this study taken mainly from Pojmański (1998) and Budding \& Zeilik (1987)

\begin{tabular}{lllll}
\hline Parameter & SV Cam & XY UMa & HD 42807 & HD 24451 \\
& & & \\
\hline & & & \\
Spectral Type & F5 + K0V & G0V+K5V & G2V & K4V \\
Time of Conjunction $T_{\mathrm{hjd}}$ & 2249350.3037 & 2449350.6726 & & \\
Orbital Period $P_{\text {orb }}($ day $)$ & 0.593071 & 0.47899493 & \\
Primary Radius $R_{1}\left(R_{\odot}\right)$ & 1.11 & 1.01 & \\
Secondary Radius $R_{2}\left(R_{\odot}\right)$ & 0.74 & 0.52 & \\
Systemic Velocity $\gamma\left(\mathrm{km} \mathrm{s}^{-1}\right)$ & -13.7 & -10.5 & \\
Primary Semi-amplitude $K_{1}\left(\mathrm{~km} \mathrm{~s}^{-1}\right)$ & 118.5 & 122.5 \\
Secondary Semi-amplitude $K_{2}\left(\mathrm{~km} \mathrm{~s}^{-1}\right)$ & 211.5 & 202.0 & \\
$a_{1} \sin i\left(R_{\odot}\right)$ & 1.39 & 1.16 & \\
$a_{2} \sin i\left(R_{\odot}\right)$ & 2.48 & 1.91 & \\
$v \sin i\left(\mathrm{~km} \mathrm{~s}^{-1}\right)$ & 102 & 110 & \\
& & & \\
\hline
\end{tabular}

WHT revealed possible spicule emission, low-lying plagelike material and highly turbulent extended absorption features. These studies have demonstrated that eclipsing active binaries are a powerful tool for studying coronal and chromospheric properties over a range of rotation periods, spectral types and luminosity classes. The results to date complement the work done on highly active single stars such as AB Dor and HK Aqr.

Questions raised by studies to date include (i) are prominence-like features common in RS CVn systems as well as single active dwarfs? (ii) what are the similarities and differences between such features observed in single and binary stars and in stars of different spectral type, luminosity class and rotation rate? (iii) what are the physical properties and evolutionary time-scales of prominences and related phenomena? (iv) what are the implications of cool condensations in the coronal environment? (v) do coronal formations play a role in the mass loss process and are CME events a contributor to the overall angular momentum evolution of stars? and (vi) what is the contribution of mass loss to the ISM?

In this paper we present the results of high-resolution spectroscopic observations of two short period RS CVn stars, SV Cam and XY UMa. The aim of the observations was to search for excess emission and absorption features which could be attributed to specific activity phenomena in these binaries. In Sect. 2 we briefly summarise the characteristics of our program stars, in Sect. 3 we discuss the observations and the spectral subtraction technique. In Sect. 4 we present the results of our data analysis and discuss the significance of the results.

\section{Target stars}

The parameters of both target and standard stars used in this study are given in Table 1 .

SV Camelopardalis (HD 44982, BD+82 $174, \mathrm{HIC}$ 32015) belongs to the short-period group of RS CVn binaries (Hall 1976). Since discovered as a variable star by Guthnick (1929), SV Cam has been observed by Wood (1946), Van Woerden (1957), Hilditch et al. (1979) and Patkos (1982). The light curves have been analysed by Zeilik et al. (1988), Budding (1977), Budding \& Zeilik (1987) and Djurašević (1998), using spot modelling techniques. According to these authors, a single active darkspotted region covers a significant part of the stellar surface at high latitudes and is responsible for the migration waves which tend to fall into two longitude sectors, $45^{\circ}-135^{\circ}$ and $225^{\circ}-315^{\circ}$. Djurašević (1998) claimed that a double spot model fits the observations better than the previously used single spot model.

A multi-wavelength campaign of optical and X-ray observation of SV Cam was performed by Hempelmann et al. (1997). The total X-ray output of the SV Cam binary was found to be on a level typical for a single main sequence star. Thus, SV Cam does not appear to be overactive, contrary to what is generally claimed for some RS CVn systems. Hempelmann et al. (1997) modelled the X-ray light curve with two extended coronal emission regions, one located above a photospheric region (a spot) on the primary and the second source located between the two components of the binary. Their Doppler images suggested a spot was located on the primary star centered at a stellar latitude of $60^{\circ}$. The second Doppler source correlated well with the second X-ray source. Furthermore, they found evidence of strong activity on the secondary star of SV Cam by analysing the strength of the $\mathrm{H} \alpha$ absorption line during the secondary eclipse.

XY Ursae Majoris (HD 237786, BD+55 ${ }^{\circ} 1317$, HIC 44998) was first noted by Greyer et al. (1955) as an eclipsing binary. Since then it has been observed in the optical (Greyer 1980; Zeilik et al. 1982; Jassur 1986; Heckert \& Zeilik 1988; Qisheng et al. 1989; Zeilik et al. 1988; Hilditch \& Bell 1994; Jeffries et al. 1995; Collier Cameron \& Hilditch 1997; Erdem \& Güdür 1998), the radio (Drake et al. 1986; Morris \& Mutel 1988), the infrared 
(Arévalo \& Lázaro 1990; Mitrou et al. 1996), the ultraviolet (Greyer \& Hoffmann 1981) and the X-ray (Bedford \& Greyer 1986; Bedford et al. 1990; Dempsey et al. 1993).

The spectroscopic elements for XY UMa were derived by Rainger et al. (1991), Pojmański \& Udalski (1997) and Pojmański (1998). Pojmański \& Udalski (1997) observed transient absorption features drifting across the $\mathrm{H} \alpha$ line and suggested co-rotating clouds in the system. Arévalo \& Lázaro (1999) found an excess emission feature located at the primary star velocity and suggested that the asymmetry of the $\mathrm{H} \alpha$ emission indicated a contribution from the spectroscopically undetected secondary star. An extended structure between the components could be responsible for the $\mathrm{H} \alpha$ emission excess.

\section{Observations and analysis}

High resolution spectroscopic observations of SV Cam and XY UMa were obtained during a 5-night observing run from 11 to 15 February 1999, carried out with ELODIE, a fibre-fed echelle spectrograph of the Observatoire de Haute-Provence. This spectrograph has been in operation since the end of 1993 on the $1.93 \mathrm{~m}$ telescope and provides a spectral resolution of $\frac{\lambda}{\Delta \lambda}=45000$ throughout the visible part of the spectrum (between 3900 and $6800 \AA$ ). An automatic data reduction procedure provides data which are wavelength calibrated and flux corrected. A description of the instrument is given by Barrane et al. (1996).

Observations were timed to provide complete orbital phase coverage for both target stars. Phases were subsequently calculated according to the ephemerides given in Table 1. In total, 85 spectra for SV Cam and 69 spectra for XY UMa were obtained with 15 min integration times over the range 3906 to $6811 \AA$. For the spectral subtraction procedure, the "standard stars" were HD 42807 and HD 24451. These stars should be similar to the program star in all respects other than the level of chromospheric activity. During our observations, all five nights varied in quality and in some instances suffered from poor seeing and intermittent clouds. Thus the quality of the data was highly variable with $\mathrm{S} / \mathrm{N}$ values as high as 50 but as low as 7 in the worst case.

As shown by Gunn \& Doyle (1997) and earlier by Barden (1984) spectral subtraction provides a technique of studying stellar activity by isolating that part of the spectral signature due to chromospheric activity. This involves simulating the inactive spectrum of the star concerned and performing a linear subtraction of this from the observed spectrum. Any manifestation of activity is then visible in the subtracted spectrum as emission or absorption features. Relative changes in these features can then help to determine the time behaviour and spatial locations of both on-disk and off-disk features. To simulate the correct spectrum representing the inactive contribution, standard stars which closely match the spectral type, surface gravity and chemical composition of the binary components are observed. These spectra are then combined with the correct radial velocities, rotational velocities and intensity weights to match the spectrum of the observed active binary (Gunn 1995; Gunn \& Doyle 1997). The practical implementation of this technique suffers from several problems, particularly for binary systems such as those studied in this work. A major mismatch in spectral types between the inactive and active stars could manifest itself as a concavity or convexity in lines of interest due to the $T_{\text {eff }}$ dependence of line wings (we should return to this point below). Also, if the target star line profiles are dominated by Doppler broadening due to high rotation rates and observations are performed close to binary conjunction then resolution of the individual components may be extremely difficult.

Several questions may be raised concerning the spectral subtraction technique. Firstly, is the representation of an inactive spectrum a true depiction of the basal state of the photosphere and inactive chromosphere? Secondly, is the linear subtraction of an inactive spectrum justified for a regime where highly non-linear radiation transfer takes place? A full discussion of these issues was presented by Gunn \& Doyle (1997) who conclude that, although spectral subtraction underestimates the degree of chromospheric activity, in very active stars these assumptions create negligible errors. The derived excess emission or absorption features fairly well characterise the chromospheric behaviour.

The implementation of the spectral subtraction technique for this work is similar to that discussed by Barden (1984). However, we do not use an iterative matching technique to derive radial and rotational velocities and intensity weights due to the high degree of line blending and because we observe throughout conjunctions. We have used the CORREL code described by Gunn (1995) and Gunn \& Doyle (1997). Traditionally, intensity weights for spectral subtraction are obtained from photometric light-curves which are not representative of the light ratio during eclipses. We therefore compute intensity weights using the assumed component radii and the values of the Planck function for each effective temperature at the spectral order central wavelength. The effective temperatures are computed using the standard spectral typetemperature relations given by Schmidt-Kaler (1982). During eclipses an obscuration correction is applied to the intensity weights. Details of the procedure for combining standard spectra are given by Gunn \& Doyle (1997).

Experiences with CORREL have shown that a slight mismatch between the spectral type of the primary's template and the primary itself (e.g. in the case of SV Cam) does not cause any major problems. This is because the code finds the velocity curves for the two components and the FFT technique buried in the code is extremely powerful in pulling the two spectra out of the blended lines. The result is that the code will use its secondary template to find the secondary component spectrum and it is relatively important how well the other spectrum matches the primary. Hence, errors in the final subtracted spectra are dependent only on mismatches between the individual templates, and not on their combination. This means that 

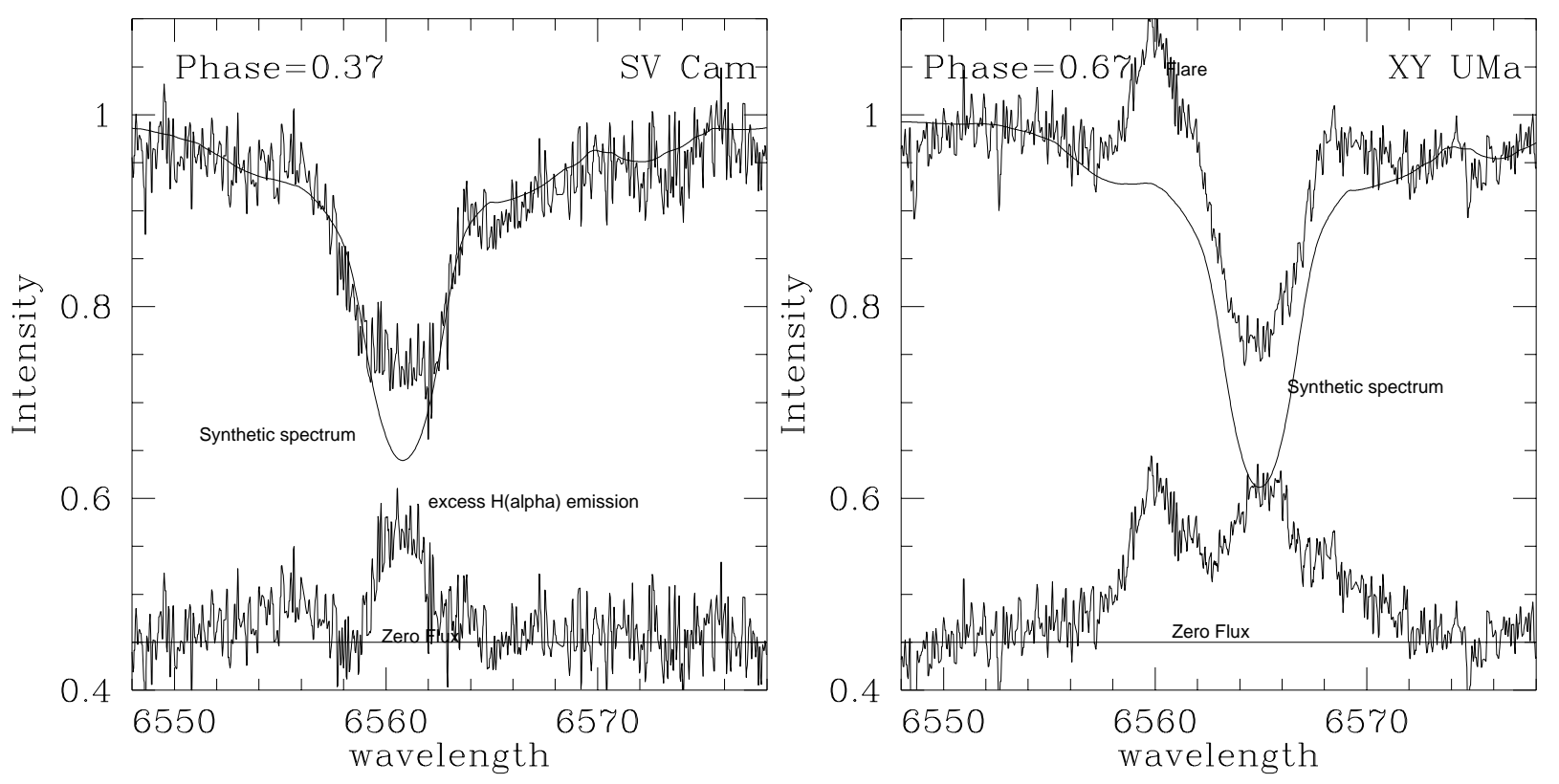

Fig. 1. Examples of the observed $\mathrm{H} \alpha$ region, the synthetic spectra and subtracted spectra for SV Cam and XY UMa. The resulting subtracted spectra show excess emission for both stars. A flare-like event is seen on the left side of the excess H $\alpha$ emission from XY UMa. Note that the bottom panel has been arbitrarily shifted in flux

the analysis of features on the secondary component won't be greatly affected by the primary spectrum mismatch.

\section{Results and discussion}

For both our target stars we performed spectral synthesis for the $\mathrm{H} \alpha$ and $\mathrm{H} \beta$ spectral orders. Figure 1 shows an example of the observed $\mathrm{H} \alpha$ region plus the synthetic and subtracted spectra for both SV Cam and XY UMa. We found that both stars displayed varying excess absorption and emission components.

\subsection{SV Cam}

Although the quality of the present data was highly variable, we obtained complete phase coverage for SV Cam. Figure 2 a shows the observed $\mathrm{H} \alpha$ line profiles versus phase while Fig. 2b shows the excess emission/absorption (synthesised) spectra versus phase. Between phases 0.69 and 0.74 a deep absorption feature (see Fig. 3) is clearly present. However, the next day, this feature is not present although other absorption structures are present (Fig. 2).

Gaussian profiles were fitted to every distinct emission or absorption feature in the subtracted spectrum using the STARLINK package DIPSO (Howarth et al. 1996). The radial velocities of these excess emission/absorption features are plotted versus phase in Fig. 4. The main excess emission feature is clearly associated with the secondary star. It is probably an active structure rotating in-and-out of view whilst it is obviously obscured during the secondary eclipse. The large absorption feature seen at phase 0.7 seems to be associated with the primary star but may consist of two components centred around the primary star velocity. On-the-other-hand, it could be residual absorption due to the slight mismatch between target and synthetic spectra. Based on our extensive experience of the spectral subtraction technique, we estimate that model spectra give an indication of activity levels to within about $10 \%$. With precisely matched templates the errors are probably less than $1 \%$. Since the difference in activity level between spectral types F5V and G2V are small, the associated errors for the SV Cam primary should be limited only by the technique itself and not by the template spectra.

Buzasi (1989) studied the theoretical ratio of $\mathrm{H} \alpha$ to $\mathrm{H} \beta$ equivalent width using two simple geometries for active regions around late-type sub-giants and dwarfs. The first model was a vertically oriented slab of material analogous to a solar prominence illuminated by the photosphere on both sides while the second was a horizontally oriented slab analogous to a solar plage illuminated only from beneath. This study concluded that it was difficult to differentiate between plages and prominences solely on the basis of the $E W_{\mathrm{H} \alpha} / E W_{\mathrm{H} \beta}$ ratio but plage region ratios were typically $10-20 \%$ smaller than those for prominences. Low ratios $(\sim 1-2)$ can be achieved in both plage-like and prominence-like structures viewed against the stellar disk but values of $\sim 3-5$ can only be achieved in prominences seen off the stellar limb. Similar conclusions concerning the $E W_{\mathrm{H} \alpha} / E W_{\mathrm{H} \beta}$ ratio from quiescent solar prominences were given by Heasley \& Mihalas (1976) who presented a theoretical formulation of the problem. There has been a good deal of observational evidence that these conclusions apply in the solar case (Tandberg-Hanssen 1967; Landman \& Mongillo 1979; Chester 1991).

For SV Cam the equivalent widths of the excess $\mathrm{H} \alpha$ and $\mathrm{H} \beta$ lines, as well as the $E W_{\mathrm{H} \alpha} / E W_{\mathrm{H} \beta}$ ratio are shown in Fig. 5. $E W_{\mathrm{H} \alpha}$ shows the lowest level during primary 

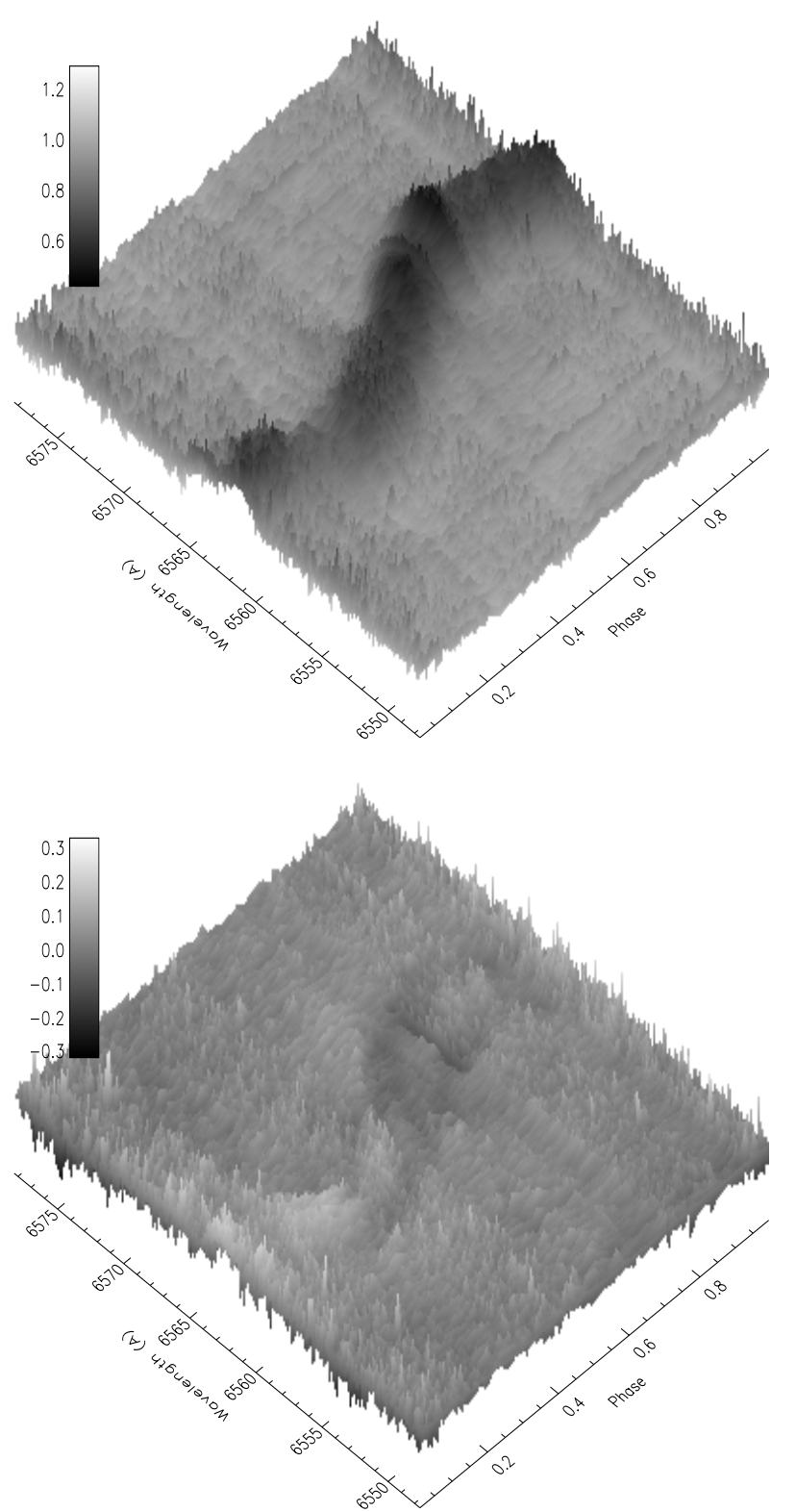

Fig. 2. a) $\mathrm{H} \alpha$ line versus phase for SV Cam and b) the excess emission in $\mathrm{H} \alpha$

eclipse and then gradually increases up to phase 0.6 before decreasing once again. $E W_{\mathrm{H} \beta}$ shows a similar, although not as pronounced, variability. The $E W_{\mathrm{H} \alpha} / E W_{\mathrm{H} \beta}$ ratio never exceeds a value of 2 which suggests the emission is due to plage regions or prominences viewed against the stellar disk.

These results seem to be consistent with the view that SV Cam is not a particularly active member of the RS CVn group. Hempelmann et al. (1997) reached a similar conclusion based on the total ROSAT X-ray flux measured from the system. The same authors concluded, on the basis of optical spectroscopy, that the secondary star in SV Cam shows a completely filled-in $\mathrm{H} \alpha$ line due to an active chromosphere. We have found that subtracted spectra display obvious excess emission from the secondary component in agreement with this result. The K0V star in
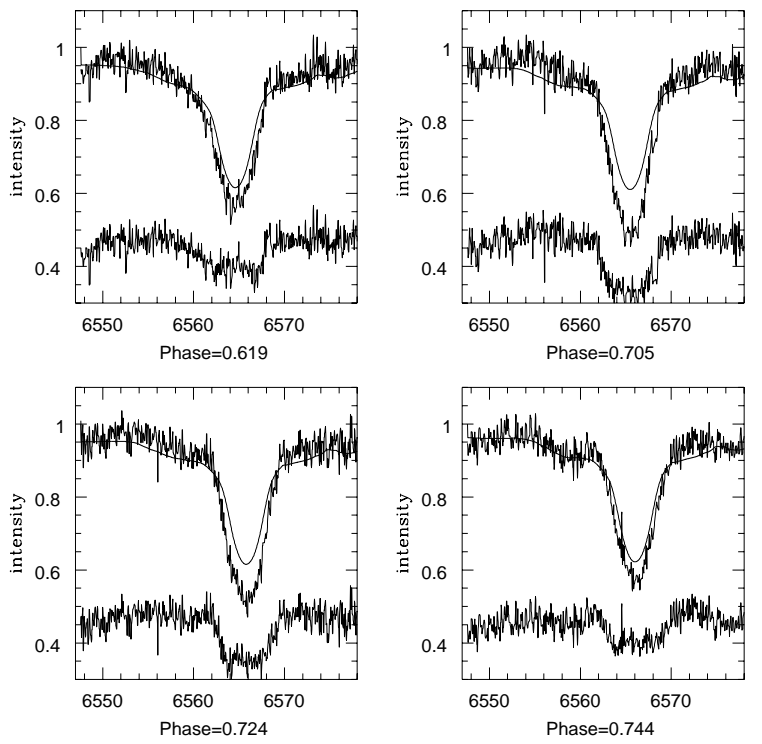

Fig. 3. The $\mathrm{H} \alpha$ region of SV Cam at around phase 0.7; the top panel shows the synthetic spectrum as a solid line while the bottom panel is the resulting subtracted spectrum

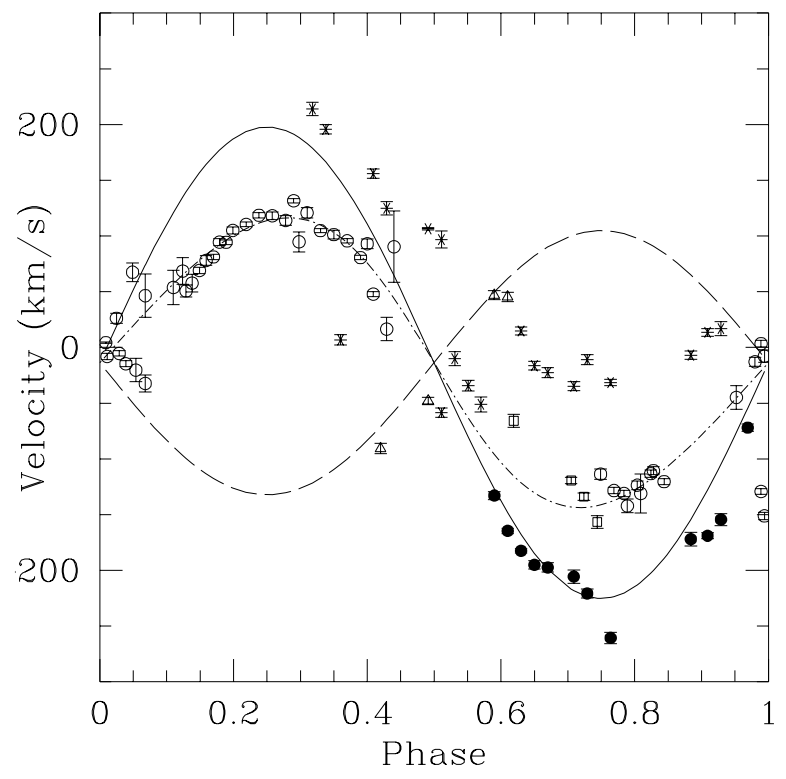

Fig. 4. The radial velocity (from Pojmański 1998) of the primary (dashed line) and secondary (solid line) versus phase for SV Cam. Superimposed is the location of the main excess emission feature (open circles), plus (open squares) absorption feature seen at phase 0.7 (see text), the open triangles, stars and filled circles show a host of other absorption features

SV Cam is thus very active although the radiation from the system is usually completely dominated by the hotter component. Furthermore, based on $E W_{\mathrm{H} \alpha}$ measurements and $\mathrm{H} \alpha$ line shape analysis, Hempelmann et al. (1997) also conclude that the secondary star displays an inhomogeneous distribution of chromospheric plage. The results of our analysis also indicate substantial plage emission from the K0V component. We find no evidence of plage regions associated with the primary component, even though the primary is known to have frequent and 


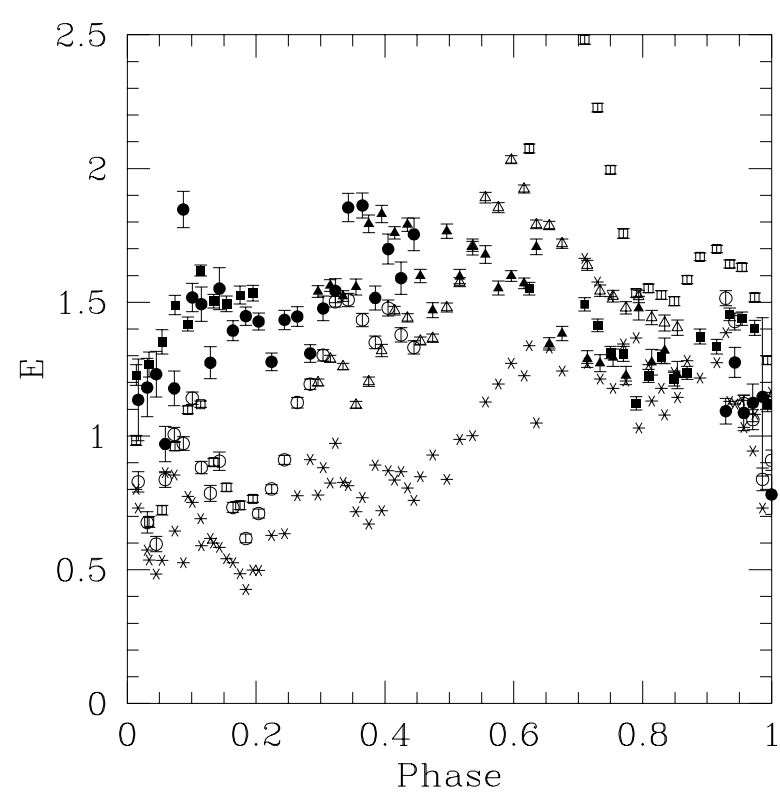

Fig. 5. Equivalent width for $\mathrm{H} \alpha$ (open symbols), and $\mathrm{H} \beta$ (closed symbols) in SV Cam. Data for the individual nights are given as $\bigcirc$ (11 Feb. 1999), $\sqcap$ (12 Feb. 1999), and $\triangle(13$ Feb. 1999). The $E W_{\mathrm{H} \alpha} / E W_{\mathrm{H} \beta}$ ratio is given as $\star$

variable surface spots, nor do we find evidence of any extended prominence-like features on either star.

An approximate calculation can be performed for the volume of the emitting region for $\mathrm{H} \alpha$ from SV Cam. The method follows that of Gunn \& Doyle (1997) and uses the atmospheric models of Kurucz (1979). Taking a log $g$ of zero (relative to the Sun) and assuming an effective temperature of $5500 \mathrm{~K}$ (the lowest value for which the models are calculated), the flux density at $\mathrm{H} \alpha$ (in fact at $6575 \AA$ ) is $1.910^{6} \mathrm{erg} \mathrm{s}^{-1} \mathrm{~cm}^{-2} \AA^{-1}$. This will be a lower limit because of the lower effective temperature of the secondary component of SV Cam. Assuming a radius for this star of $0.74 R_{\odot}$, the mean equivalent width of the excess $\mathrm{H} \alpha$ emission is $1.32 \AA$ measured over approximately $2 \AA$ across the $\mathrm{H} \alpha$ line. The resulting excess emission luminosity is then $2.110^{29} \mathrm{erg} \mathrm{s}^{-1}$. The volume emissivity of $\mathrm{H} \alpha$ is given by;

$j=1.2910^{-23} n_{\mathrm{e}}^{2} T^{-1 / 2} \log \left\{\frac{I_{\mathrm{H}}}{k T}\right\}$

where $I_{\mathrm{H}}$ is the ionization energy of hydrogen. The region of formation of the $\mathrm{H} \alpha$ core lies within an electron density of $3.510^{10}$ to $110^{11}$ and an electron temperature of 6000 to $10000 \mathrm{~K}$. The corresponding range in the volume of the emission region is $8.810^{31}-1.310^{32} \mathrm{~cm}^{3}$. Assuming the emission region is a homogeneous hemispherical shell then the chromospheric thickness for $\mathrm{H} \alpha$ lies in the range $0.07-$ $0.09 R_{\odot}$. These values are likely to be overestimated due to the higher effective temperature taken for the atmospheric models. However, this range is consistent with chromospheric thicknesses derived by Gunn \& Doyle (1997) and Gunn et al. (1997). In general the chromospheric thicknesses of RS CVns are much higher than those observed in the Sun (Athay 1971; Fraquelli 1984).
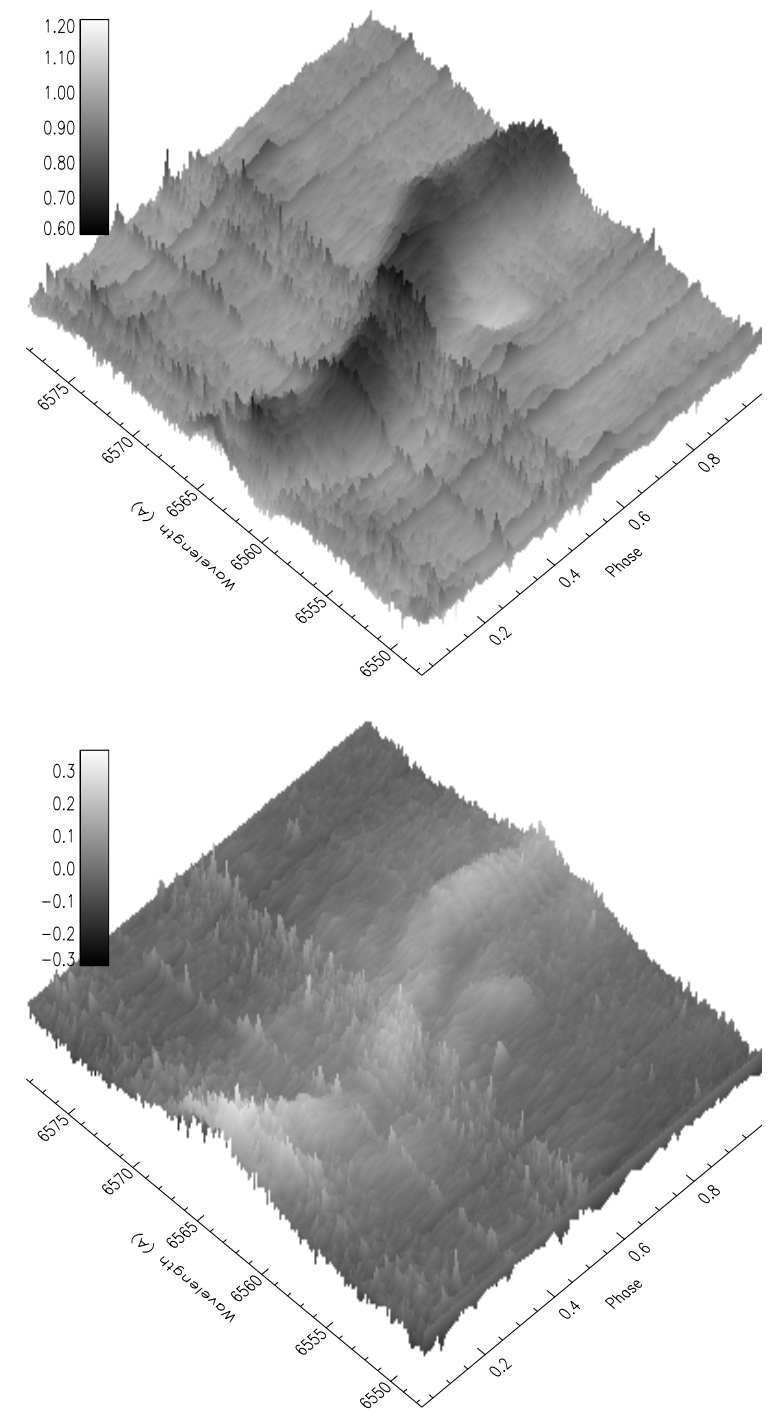

Fig. 6. a) $\mathrm{H} \alpha$ line versus phase for $\mathrm{XY} U M a, b)$ the excess emission in $\mathrm{H} \alpha$

\section{2. $X Y \cup M a$}

Figure $6 \mathrm{a}$ shows the observed $\mathrm{H} \alpha$ profile versus phase while Fig. 6b shows the emission excess. No obvious absorption structures are present during these observations. As in SV Cam, Gaussian profiles were fitted to every distinct emission or absorption feature in the subtracted spectra. Figure 7 shows the radial velocities of these features against orbital phase. Just as in SV Cam, the main excess emission feature is clearly associated with the secondary star. It is also probably an active structure rotating in-and-out of view. Since it is not eclipsed by the primary component it must be concentrated on the hemisphere of the star that is not obscured during the eclipse. During the observations, between phases 0.6 and 0.8 , we observed a flare-like event which lasted for more than two hours. From the radial velocity curve, this event occured close to the primary, although not on its surface. We do not present a full analysis of this event in this paper. 


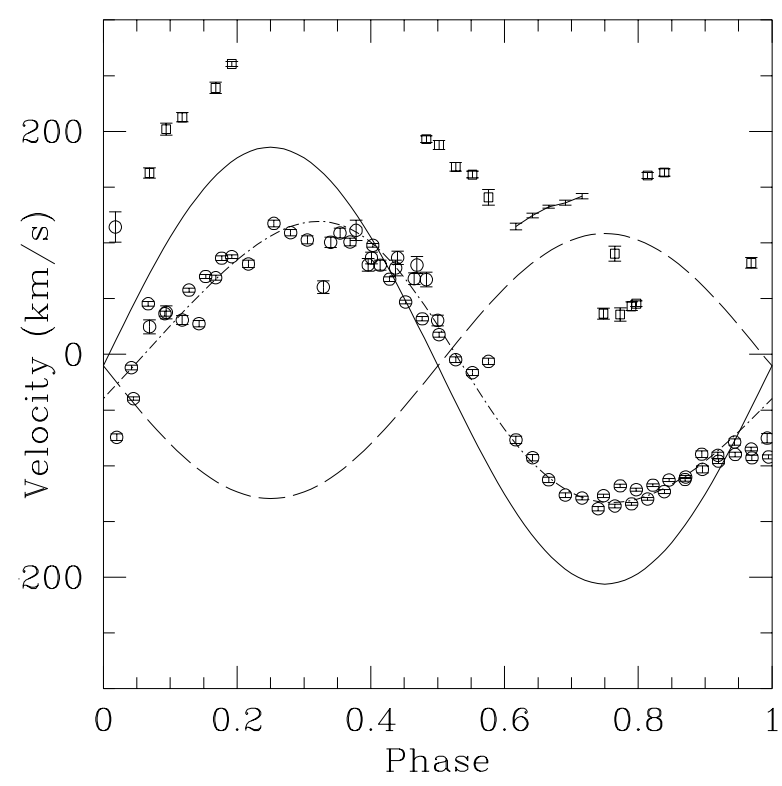

Fig. 7. The radial velocity (from Pojmański 1998) of the primary (dashed line) and secondary (solid line) versus phase for XY UMa. Superimposed is the location of the main excess emission (open circles). The other symbols show the other emission structures located to the side of the central emission feature

For XY UMa the equivalent widths of the excess $\mathrm{H} \alpha$ and $\mathrm{H} \beta$ lines, as well as the $E W_{\mathrm{H} \alpha} / E W_{\mathrm{H} \beta}$ ratio are shown in Fig. 8. Some variability is seen in $E W_{\mathrm{H} \alpha}$ and $E W_{\mathrm{H} \beta}$, although no definitive statements can be made concerning the cause. Once again, the $E W_{\mathrm{H} \alpha} / E W_{\mathrm{H} \beta}$ ratio never exceeds a value of 2 which suggests the emission on the secondary is due to plage regions or prominences viewed against the stellar disk. There is no evidence for extended structures surrounding either component of the system.

Arévalo \& Lázaro (1999) made a similar study of the $\mathrm{H} \alpha, \mathrm{H} \beta$ and $\mathrm{Ca}$ II IRT lines from XY UMa. They found that during low resolution observations taken in 1989 an asymmetric excess emission ocurred in the $\mathrm{H} \alpha$ line was primarily associated with the primary star. Subsequent high resolution spectroscopy showed that a component of the $\mathrm{H} \alpha$ emission could be associated with the secondary star. In our excess results we find no evidence of significant emission from the primary star, somewhat contrary to the results of Arévalo \& Lázaro (1999). We conclude that during our observing run, plage-like regions were extensive on the secondary component but were almost absent on the primary star. Our results for the excess emission $E W_{\mathrm{H} \alpha} / E W_{\mathrm{H} \beta}$ ratio are consistent with those of Arévalo \& Lázaro (1999).

Assuming a radius for the secondary star in XY UMa of $0.52 R_{\odot}$ and using the mean equivalent width of the excess $\mathrm{H} \alpha$ emission of $0.82 \AA$ measured over approximately $2 \AA$ across the $\mathrm{H} \alpha$ line we find that the excess emission luminosity is $5.110^{28} \mathrm{erg} \mathrm{s}^{-1}$. Using the same assumptions as for SV Cam we find that the range in chromospheric thickness for $\mathrm{H} \alpha$ lies in the range $0.22-0.38 R_{\odot}$. Again, these values are likely to be overestimated due

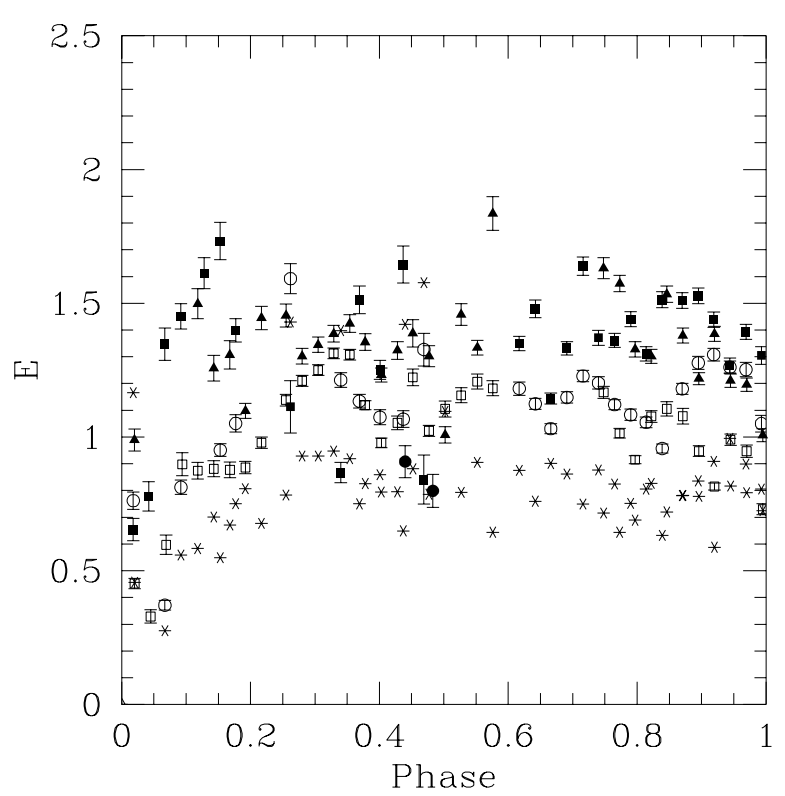

Fig. 8. Equivalent width for $\mathrm{H} \alpha$ (open symbols), and $\mathrm{H} \beta$ (closed symbols) in XY UMa. Data for the individual nights are given as $\bigcirc$ (11 Feb. 1999), $\sqcap$ (14 Feb. 1999), and $\triangle(15$ Feb. 1999). The $E W_{\mathrm{H} \alpha} / E W_{\mathrm{H} \beta}$ ratio is given as $\star$

to the higher effective temperature taken for the atmospheric models. The chromospheric thickness reported for the Sun (Athay 1971) is of the order of $0.004 R_{\odot}$ while Fracquelli (1984) showed that the two components of the RS CVn system HR 1099, have chromospheric thicknesses of $0.2 R_{\odot}$ and $0.06 R_{\odot}$ respectively. Values of $0.045-$ $0.42 R_{\odot}$ were found for ER Vul, $0.052-0.56 R_{\odot}$ for GK Hya and $0.25-1.57 R_{\odot}$ by Gunn \& Doyle (1997) and Gunn et al. (1997). The range of values derived here for the secondary component of XY UMa are therefore in the upper range previously seen in active close binaries. This confirms that the secondary component is highly active but that the emission may be arising in regions where the electron density and temperature are much higher than assumed.

\section{Conclusions}

We have analysed high-resolution spectroscopic observations of the eclipsing RS CVn-type active close binaries SV Camelopardalis and XY Ursae Majoris. Using the spectral subtraction technique we have isolated that part of the $\mathrm{H} \alpha$ and $\mathrm{H} \beta$ profiles due to the active chromospheres. For both systems we have found excess emission components in both Balmer lines. In both cases the excess emission is associated with the secondary, cooler component of the system. Analysis of the $E W_{\mathrm{H} \alpha} / E W_{\mathrm{H} \beta}$ ratio indicates that these excess emission components are probably associated with surface plage regions or prominences viewed against the stellar disks. We find no firm evidence of excess absorption features associated with extended prominencelike material in these systems. Simple calculations reveal that the chromospheric thicknesses derived from the 
excess emission is within the range usually found for active close binary stars.

Acknowledgements. Research at Armagh Observatory is grantaided by the N. Ireland Dept. of Culture, Arts and Leisure, while support for software and hardware is largely provided by the STARLINK Project which is funded by the UK PPARC. This work was supported in part by a grant (PPA/G/S/1997/00298) from the UK PPARC. FFÖ wished to thank TÜBİTAK, the Scientific and Technical Research Council of Turkey, for a grant which enabled a visit to Armagh Observatory. This research has made use of the Simbad database, operated at CDS, Strasbourg, France.

\section{References}

Arévalo, M. J., \& Lázaro, C. 1990, AJ, 99, 983

Arévalo, M. J., \& Lázaro, C. 1999, AJ, 118, 1015

Athay, R. G. 1971, in ed. C. J. Marcis, Physics of the Solar Corona (Reidel: Dordrecht), 36

Banks, T., \& Budding, E. 1989, IAU IBVS, No. 3304

Barden, S. C. 1984, Ph.D. Thesis, Pennsylvania State University

Baranne, A., Queloz D., Mayor, M., et al. 1996, A\&AS, 119, 373

Bedford, D. K., \& Greyer, E. H. 1986, Mitteil. Astron. Gesellschaft, 67, 305

Bedford, D. K., Jeffries, R. D., Greyer, E. H., \& Vilhu, O. 1990, MNRAS, 243, 557

Budding, E. 1977, Ap. Space Sci., 48, 207

Budding, E., \& Zeilik, M. 1987, ApJ, 319, 827

Buzasi, D. L. 1989, Ph.D. Thesis, Pennsylvania State University

Byrne, P. B., Eibe, M. T., \& Rolleston, W. R. J. 1996, A\&A, $311,651 \mathrm{~B}$

Chester, M. M. 1991, Ph.D. Thesis, Pennsylvania State University

Collier Cameron, A., \& Robinson, R. D. 1989, MNRAS, 236, 57

Collier Cameron, A., Duncan, D. K., Ehrenfreund, P., et al. 1990, MNRAS, 247, 415

Collier Cameron, A., \& Woods, J. A. 1992, MNRAS, 258, 360

Collier Cameron, A., \& Hilditch, R. W. 1997, MNRAS, 287, 567

Dempsey, R. C., Linsky, J. L., Fleming, T. A., \& Schmitt, J. H. M. M. 1993, ApJS, 86, 599

Doyle, J. G., \& Collier Cameron, A. 1990, MNRAS, 244, 291

Drake, S. A., Simon, T., \& Linsky, J. L. 1986, AJ, 91, 1229

Djurašević, G. 1998, A\&AS, 127, 233

Erdem, A., \& Güdür, N. 1998, A\&AS, 127, 257

Fracquelli, D. A. 1984, ApJ, 276, 243

Greyer, E. H., Kippenhahn, R., \& Strohmeier, W. 1955, Kleine Veroffentl. Remeis Sternwarte Bamberg, No. 9

Greyer, E. H. 1980, in Close Binary Stars: Observations and
Interpretations, ed. J. M. Plavec, D. M. Papper, \& R. K. Ulrich, 423

Greyer, E. H., \& Hoffmann, M. 1981, Mitteil. Astron. Gesellschaft, 52, 70

Gunn, A. G. 1995, Ph.D. Thesis, Queen's University of Belfast

Gunn, A. G., \& Doyle, J. G. 1997, A\&A, 318, 60

Gunn, A. G., Doyle, J. G., \& Houdebine, E. R. 1997, A\&A, 319, 211

Guthnick, P. 1929, Astr. Nach., 258, 83

Hall, J. C., \& Ramsey, L. W. 1992, A\&A, 318, 60

Hall, J. C., Huenemoerder, D. P., Ramsey, L. W., \& Buzasi, D. L. 1990, ApJ, 358, 610

Hall, D. S. 1976, in ed. W. S. Fitch, Multiple Periodic Veriable Stars, IAU Coll., 29 (Reidel: Dordrecht), 287

Heasley, J. N., \& Mihalas, D. 1976, ApJ, 205, 273

Heckert, P., \& Zeilik, M. 1988, IAU IBVS, No. 304

Hempelmann, A., Hatzes, A. P., Kürster, M., \& Patkós, L. 1997, A\&A, 317, 125

Hilditch, R. W., Harland, D. M., \& McLean, B. J. 1979, MNRAS, 187, 797

Hilditch, R. W., \& Bell, S. A. 1994, MNRAS, 267, 1081

Houdebine, E. R., Foing, B. H., \& Rodono, M. 1990, A\&A, 238,249

Howarth, I. D., Murray, J., Berry, D. S. 1996, DIPSO A friendly Spectrum Analysis Program STARLINK User Note 50.19

Jassur, D. M. Z. 1986, Ap\&S, 128, 369

Jeffries, R. D., Collins, C., Elliot, K. H., Pittard, J. M., \& Taylor, S. B. 1995, IAU IBVS, No. 4277

Kurucz, R. L. 1979, ApJS, 40, 1

Landman, D. A., Mongillo, M. 1979, ApJ, 230, 581

Mitrou, C. K., Doyle, J. G., Mathioudakis, M., \& Antonopoulou, E. 1996, A\&AS, 115, 61

Morris, D. H., \& Mutel, R. L. 1988, AJ, 95, 204

Mullan, D. J., Sion, E. M., Bruhweiler, F. C., \& Carpenter, K. G. 1989, ApJ, 339, L33

Patkos, L. 1982, Comm. Konkoly Obs., No. 80

Pojmański, G. 1998, Acta Astr., 48, 711

Pojmański, G., \& Udalski, A. 1997, Acta Astron., 47, 451

Rainger, P. P., Hilditch, R. W., \& Edwin, R. P. 1991, MNRAS, 248, 168

Qisheng, L., Xiaoyu, Z., \& Rhongxian, Z. 1989, IAU IBVS, No. 3374

Schmidt-Kaler, T. 1982, in Landholt-Bornstein, vol. 2b, ed. K. Schaifers, \& H. H. Voig (Springer: Heidelberg), 451

Strassmeier, K. G., Hall, D. S., Fekel, F. C., \& Scheck, M. 1993, A\&AS, 100, 173

Tandberg-Hanssen, E. 1967, Solar Activity (Waltham, MA: Blaisdell)

Van Woerden, H. 1957, Ann. Sterrew. Leiden, 21, 3

Wood, F. B. 1946, Princeton Obs. Control, No. 21

Zeilik, M., De Blasi, C., Rhodes, M., \& Budding, E. 1988, ApJ, 332, 293

Zeilik, M., Batuski, D., Elston, R., Schmolke, P., \& Smith, P. 1982, IAU IBVS, No. 2169 